\section{Allergy, immunity, and infection}

\section{G155 'SECKEL' PHENOTYPE, RADIOSENSITIVITY, GROWTH FAILURE, MYELODYSPLASIA AND COMBINED IMMUNODEFICIENCY DUE TO DNA LIGASE IV MUTATIONS}

A.R. Gennery, A.J. Cant, J. Seidel', S.E. Palmer ${ }^{2}$, M. O'Driscoll' ${ }^{3}$ K. Cerosaletti ${ }^{4}$, B. Gruhn', R. Varon ${ }^{5}$, R.A. Gatti ${ }^{4}$, B. Hirsch', P. Concannon ${ }^{4}$, P. Jeggo ${ }^{3}$. University of Newcastle upon Tyne, ${ }^{3}$ University of Sussex, UK; 'Friedrich-Schiller-University, ${ }^{5}$ Charite Humboldt University, Germany; Universities of ${ }^{2}$ Texas, ${ }^{4}$ Washington, ${ }^{6}$ California, ${ }^{7}$ Minnesota, USA

Introduction: DNA repair pathway defects cause immunodeficiency, developmental delay, lymphoreticular malignancy, e.g. Ataxia telengectasia, Niimegen Breakage Syndrome (NBS). We describe clinical characteristics of 4 patients with DNA Ligase IV (LigIV) defects.

Patients: Two male, 2 female patients (age 1-49 years), including 2 siblings. All had 'Seckel-like' appearance; growth retardation, learning difficulties and global developmental delay. Three developed pancytopenia and marrow hypoplasia. Two had panlymphopenia with profound B cell lymphopenia. T cell responses to lymphocyte mitogens were markedly reduced. Immunoglobulin levels were normal, but lgG2 subclass was below the age-related reference. Specific antibody response to protein antigens were normal, pneumococcal polysaccharide antigen response was impaired. One patient had cryptorchism, hypogonadism, early tooth loss, hypothyroidism and type 2 diabetes mellitus. One developed skin photosensitivity, hypothyroidism, abnormal menses and psoriasis. One developed multiple psoriasiform erythrodermic and squamous skin patches. Additionally, skeletal radiographs revealed scoliosis, abnormal rib shape, hypoplastic pelvis, splayed metacarpals with pseudoepiphyses, atypical bone maturation, and 5th finger clinodactyly.

Laboratory Findings: Fibroblast radiosensitivity was similar to NBS cells. A homozygous LiglV mutation resulted in impaired LigIV function in one. The siblings are compound heterozygotes for two LiglV truncating mutations, causing low residual protein expression and impaired XRCC4 and LigIV interaction. The final patient has compound LiglV heterozygous mutations causing impaired function.

Conclusion: LiglV mutations should be considered in patients with Seckel appearance, marrow hypoplasia and combined immunodeficiency. Phenotype appears to correlate with the severity of LigIV protein dysfunction.

\section{G156 INCREASED INCIDENCE AND SEVERITY OF THE SYSTEMIC INFLAMMATORY RESPONSE SYNDROME IN PATIENTS DEFICIENT IN MANNOSE BINDING LECTIN}

K.J. Fidler', P. Wilson², J. Davies ${ }^{3}$, M. Turner ${ }^{4}$, N. Klein', M.J. Peters². 'Dept of Infectious Diseases \& Microbiology and ${ }^{4}$ Dept of Immunobiology, Institute of Child Health, London; ${ }^{2}$ Paediatric Intensive Care Unit, Great Ormond St Hospital for Children NHS Trust; ${ }^{3}$ Dept of Gene Therapy, Royal Brompton Hospital, London

Background: The Systemic Inflammatory Response Syndrome (SIRS) following surgery, infection or trauma is a major cause of morbidity and mortality on paediatric intensive care $(\mathrm{PIC})$. MBL is a serum protein that functions as a direct opsonin and causes antibody-independent complement activation. Patients with polymorphisms in the MBL-2 gene have an increased susceptibility to and severity from a number of diseases. MBL also influences cytokine production and may therefore contribute to the development of SIRS.

We hypothesized that PIC patients with MBL polymorphisms have an increased risk of developing early SIRS and subsequent organ failure compared to controls.

Method: The frequency of polymorphisms in the exon 1 part of the MBL-2 gene was compared between 2 groups of children, one group with SIRS the other without. All children recruited were part of a prospective, observational study of consecutive children admitted to PIC in a tertiary hospital. MBL genotype was determined by PCR and heteroduplex analysis.

Results: 100 cases were recruited as follows 1) Infection $n=50$ \& 2) Non infection $n=50$. These were sub divided into those with, $n=59$, and without, $n=41, S I R S$, irrespective of aetiology. Those with polymorphisms in the MBL genotype were significantly more likely to develop SIRS regardless of the type of initial insult (Fishers exact test $\mathrm{p}<0.0001$, odds ratio for development of SIRS with MBL polymorphism=7). In the infection group MBL deficiency was associated with the development of septic shock (12/16 MBL deficient with septic shock vs $2 / 15$ in the infection only group, $p=0.0007$ ).

Conclusions: $\mathrm{MBL}$ polymorphisms are associated with greatly increased risk of developing SIRS and of progression from infection to severe sepsis with organ failure in paediatric intensive care patients.

G157 INCREASED INCIDENCE OF EPSTEIN BARR VIRUS (EBV) RELATED DISEASE FOLLOWING PAEDIATRIC STEM CELL TRANSPLANT (SCT) DUE TO REDUCED INTENSITY CONDITIONING (RIC); AN EMERGING PROBLEM

M.M. Gandhi, J. Cohen, P. Naik, K. Rao, D. Cubitt, P. Amrolia, H.B. Gaspar, G. Davies, P. Veys. Great Ormond Street Hospital for Children, London WCIN 3JH, UK

Background: EBV is an oncogenic virus associated with post transplant lymphoproliferative disease (LPD). EBV LPD is uncommon following non-T cell depleted SCT using conventional intensity conditioning $(\mathrm{ClC})$. Recent reports have, however, highlighted its occurrence after reduced intensity conditioning $(\mathrm{RIC}) \mathrm{SCT}$ in adults.

Aim: Assess incidence of EBV viraemia, and associated LPD in a consecutive cohort of children undergoing RIC SCT from January 1999 and June 2002

Results: 128 patients [malignant disease (47), genetic disorder (81)] had 133 transplants. All had prospective weekly monitoring for EBV infection by quantitative PCR. 68 patients received $\mathrm{CIC}$ plus in vivo Campath $(n=38)$ or ATG ( $n=14)$; 65 patients had RIC plus in vivo Campath $(n=35)$ or ATG $(n=29)$. EBV viraemia occurred in $7 / 68$ $(10 \%)$ and $23 / 65(35 \%)$ children following $\mathrm{CIC}$ and $\mathrm{RIC}$ respectively. Following $\mathrm{CIC}, 5 / 38(13 \%)$ and $2 / 14(14 \%)$ children receiving in vivo Campath and ATG respectively developed EBV viraemia; all were asymptomatic and none developed LPD. After RIC, EBV viraemia developed in $9 / 35(26 \%)$ and $13 / 29(45 \%)$ after in vivo Campath and ATG respectively. 17/23 patients had concurrent reactivation of CMV, adenovirus or both. $8 / 23$ patients had asymptomatic EBV viraemia, 8 had associated rash and fever, and 7 developed LPD; $2 / 7$ died of EBV LPD. EBV appeared to originate from the recipient B-cell pool in 2/7 LPD patients. Specific therapy included EBV CTLs, DLI and Ganciclovir; early use of Rituximab was most efficacious.

Conclusion: There is a higher incidence of EBV-related viraemia and LPD following RIC SCT in children, particularly with ATG use. Profound immuno-suppression after RIC may explain the high concurrent reactivation rate of other viruses. Relative failure to eradicate recipient $B$ cells with $\mathrm{RIC}$ versus $\mathrm{CIC}$ may also be partly responsible. The use of Campath to remove recipient/donor B as well as $T$ cells reduces the occurence of EBV viraemia following RIC but the incidence remains high.

\section{G158 HAEMOPHILUS INFLUENZAE TYPE B (HIB) ANTIBODY} RESPONSE IN SIGNIFICANTLY PRETERM UK INFANTS

J.E. Berrington, A.C. Fenton, G.P. Spickett, A.J. Cant. Royal Victoria Infirmary, Newcastle upon Tyne, UK

Introduction: Invasive Hib disease is more common in preterm infants.' There are little data to support the efficacy of vaccinating infants $<32$ weeks against Hib at 2, 3 and 4 months of postnatal age with no booster dose. Some vaccine responses are reduced in these infants. ${ }^{2,3}$ We measured Hib antibody response after pragmatic primary vaccination.

Methods: Infants $<32$ weeks were recruited from four tertiary neonatal units. After consent blood was taken pre- and post-primary vaccination, serum separated, and stored at $-80^{\circ} \mathrm{C}$. Ig G against $\mathrm{Hib}$ was measured by ELISA (The Binding Site). Data were log transformed prior to analysis, and are presented as geometric mean titres (GMT). If post primary titre was $<1.0 \mathrm{mcg} / \mathrm{ml}$, a booster dose of Hib was offered.

Results: 134 infants with median gestation 28.1 weeks (range 23.6-31.8) and median weight $1040 \mathrm{~g}$ (range 425-2500) were studied. $57 \%$ were male, $12 \%$ had postnatal steroids. Hib antibody responses are shown in the table.

Conclusion: Significantly preterm infants have a poor $\lg G$ response to Hib vaccine when given the current routine schedule. 55\% of infants have levels not considered protective. These infants may merit a booster dose, to which they appear to respond well.

1. Heath PT et al. Clin Infect Dis 2000;31:973-80.

2. Munoz A et al. Pediatrics 1995;96:216-19

3. Slack MH et al. J Infect Dis $2001 ; 184: 1617-20$. 
Abstract $\mathrm{G} 158$

\begin{tabular}{llllll}
\hline $\operatorname{lgG}(\mathrm{mcg} / \mathrm{ml})$ & Pre $\mathrm{n}=134$ & Post $\mathrm{n}=134$ & $\%<1.0$ & $\%<0.15$ & Post boost $\mathrm{n}=34$ \\
\hline GMT $(95 \% \mathrm{Cl})$ & $0.15(0.12-0.19)$ & $0.73(0.53-1)$ & 55 & 21 & $8.16(5.8-11.45)$ \\
\hline
\end{tabular}

\section{G159 WHY IS MOTHER-TO-CHILD TRANSMISSION OF HIV INFECTION STILL OCCURRING IN THE UK AND IRELAND? REPORTED BIRTHS 1998-2002}

P.A. Tookey, J. Masters, on behalf of the HIV surveillance teams at the Institute of Child Health, London, the PHLS Communicable Disease Surveillance Centre, and the Scottish Centre for Infection and Environmental Health.

Aims: To explore the reasons why infants are still being born with HIV infection in the UK and Ireland, when antenatal testing is widespread and interventions to prevent transmission are so successful.

Methods: HIV-infected children and infants born to HIV-infected women are reported to the National Study of HIV in Pregnancy and Childhood (NSHPC) through two active reporting schemes run in collaboration with the Royal College of Obstetricians and Gynaecologists and the RCPCH's British Paediatric Surveillance Unit. Information on demographic characteristics, timing of maternal diagnosis and uptake of interventions (antiretrovirals, mode of delivery, not breastfeeding), was examined.

Results: Over 1700 British-born infants born to HIV-infected women have been reported to the NSHPC since 1998, $90 \%$ to women diagnosed prior to delivery. At the time of writing, 7\% (122) are known to be infected and $56 \%$ not infected. Almost all the infants whose infection status is not yet reported were born to diagnosed women, and very few of them are likely to be infected. $102(84 \%)$ of the infected infants were born to undiagnosed women, and 20 (16\%) to women diagnosed before delivery. All but one of the 20 diagnosed women were from Africa; diagnosis was usually close to the time of delivery, or interventions were declined, so in most cases little or no anti-retroviral therapy was taken. Among the 102 undiagnosed women, $74 \%$ were African and $20 \%$ British-born; at least $70 \%$ had vaginal deliveries and $64 \%$ breastfed their babies; $11 \%$ of their babies have died, most under the age of 12 months. A routine offer of antenatal testing was not in place at the relevant time in units where at least half of the infected infants were born.

Conclusions: Vertical transmission of HIV infection still occurs in the UK and Ireland, mainly associated with failure to diagnose infected pregnant women. Understanding the circumstances under which mother-to-child transmission is occurring at the present time, and identifying missed opportunities for preventing this, will help to further reduce transmission rates.

\section{G160 SERVICE PROVISION FOR CHILDHOOD TUBERCULOSIS (TB) IN GREATER LONDON}

M. Paliwalla', J. Moore-Gillon², V. Novelli' for the Joint Tuberculosis Committee of the British Thoracic Society. 'Infectious Diseases Unit, Great Ormond Street Hospital NHS Trust, London; ${ }^{2}$ Respiratory Medicine Department, Barts \& the London NHS Trust, London

To investigate the management of TB in children in Greater London we sent questionnaires to the most appropriate adult respiratory physician (ARP) and paediatrician in each of 26 hospital trusts. Responses were received from 47 of 51 individuals approached, all 26 trusts being represented.

Overall management was exclusively by paediatricians in 13 trusts (2 Infectious Disease [ID], 5 respiratory interest, 6 general). In 7 trusts there was shared care between paediatricians and ARP. In 3 trusts care was by an ARP alone, and in 3 trusts care was by either paediatrician or ARP. Half the trusts see fewer than 15 cases per year. In 19 trusts, one consultant had overall responsibility for most or all cases of childhood TB: 6 general paediatricians, 8 generalists with respiratory interest, 3 paediatric ID and 2 ARP. All in-patients were managed on paediatric wards, 2 trusts using paediatric ID wards. In 22 trusts in-patients were supervised by a paediatrician alone (14 general, 3 ID, 5 respiratory interest). 4 trusts managed in-patients jointly with an ARP. 5 trusts had negative pressure isolation rooms for paediatric cases. Outpatients were seen in paediatric clinics (15), joint clinics with ARP (3), ARP clinics (2), and in 6 trusts some were seen in paediatric and some in adult clinics. TB nurses were involved in the management, including contact tracing, for paediatric TB in 24 trusts.
19 trusts could provide paediatric Directly Observed Therapy within their hospitals or through local community services.

Our survey demonstrates widely varying models of care for childhood TB in London. There may be yet wider variation nationally. TB in children is relatively uncommon. Few paediatricians can gain adequate experience to ensure optimal care of the disease itself. They cannot deal with adult family cases and contacts. Conversely, adult respiratory physicians are unable to deal with the wider aspects of the child's care. A joint approach, with paediatricians and adult respiratory physicians sharing responsibility for the family unit is practised in some trusts and may be the best model.

\section{G161 CEREBROSPINAL FLUID GLUCOSE LEVELS AND SENSORINEURAL HEARING LOSS IN BACTERIAL MENINGITIS}

M. Eisenhut, T. Meehan, L. Batchelor. Royal Liverpool Childrens NHS Trust; Welsh Hearing Centre, University Hospital of Wales, Cardiff; Macclesfield District General Hospital NHS Trust

Aims: The comparison of cerebrospinal fluid (CSF) glucose levels in children with and without a sensorineural hearing loss ( $\mathrm{SNHL}$ ) following bacterial meningitis; to examine the association of CSF glucose levels with type of infecting organism, duration of symptoms before diagnosis and severity of the $\mathrm{SNHL}$.

Methods: A retrospective (1987-2000) multicentre case control study was conducted on 192 individuals with a history of bacterial meningitis of which 47 cases developed a SNHL and 145 were normally hearing controls. Centres included in the study were Macclesfield District General Hospital, St Mary's Hospital, Royal Manchester Children's Hospital, Pendlebury and Booth Hall Hospitals in Manchester and the Royal Liverpool Childrens NHS Trust (Alder Hey).

Results: The median patient age (IQR) was 12 months (21). The mean CSF glucose level (SD) was $1.3 \mathrm{mmol} / \mathrm{l}(1.2)$ in cases and $2.5 \mathrm{mmol} / \mathrm{l}(1.6)$ in controls $(p<0.001$, t-test for samples with unequal variance). A subgroup analysis of those with Streptococcus (S.) pneumoniae, Haemophilus (H.) influenzae and Neisseria (N.) meningitidis infection showed that CSF glucose levels were lower in cases with hearing loss independent of infecting organism but this was not statistically significant for N. meningitidis. The CSF glucose levels were significantly $(p<0.05)$ lower in cases independent of the duration of symptoms before diagnosis. No SNHL was observed for a symptom duration of less than 12 hours. The risk of SNHL increased with a delay in diagnosis. CSF glucose levels in patients with mild to moderate versus severe to profound and unilateral versus bilateral SNHL were not significantly different.

Conclusions: The intensity and duration of the process leading to low CSF glucose levels in bacterial meningitis is associated with an increased risk of SNHL. This is independent of the infecting organism but less so for $\mathrm{N}$. meningitidis. The severity of SNHL is not associated with CSF glucose levels.

\section{G162 UPPER RESPIRATORY TRACT CARRIAGE AND TRANSMISSION OF PNEUMOCOCCI}

M. Hussain', R. Talukdar' ${ }^{2}$, S. Martin², R. Pebody ${ }^{3}$ D. Goldblatt' ${ }^{1}$ A. Efstratiou $^{2}$, R. George 2 E. Miller'. 'Institute of Child Health \& Great Ormond Street Hospital; ${ }^{2}$ Central Public Health Laboratory, Colindale; ${ }^{3}$ Immunisation Division of the Communicable Disease Surveillance Centre, Colindale

Aim: To undertake a longitudinal carriage study of pneumococci in families prior to possible large scale introduction of the new vaccine.

Methods: 132 families with a pre-school child, were recruited from 4 GP practices across Herffordshire. 104 families remained till the end of the study; $21 \%$ withdrawal rate. Monthly nasopharyngeal swabs were taken from all household members for 10 months, starting in October 2001 (01) and completing in July 2002 (10). Basic Epidemiological data were also gathered. At the end of the study the eligible children were offered the 7-valent pneumococcal conjugate vaccine and post vaccine swab and sera were collected. 


\begin{tabular}{|c|c|c|c|c|c|c|c|c|}
\hline \multicolumn{9}{|c|}{ Abstract G162 } \\
\hline Mth & $\begin{array}{l}\text { No. of } \\
\text { swabs }\end{array}$ & $\begin{array}{l}\text { Overall } \\
\text { carriage }\end{array}$ & $\begin{array}{l}<1 \mathrm{yr} \\
(\%)\end{array}$ & $\begin{array}{l}1-2 \mathrm{yr} \\
(\%)\end{array}$ & $\begin{array}{l}2-3 \mathrm{yr} \\
(\%)\end{array}$ & $\begin{array}{l}3-5 \mathrm{yr} \\
(\%)\end{array}$ & $\begin{array}{l}5-18 \mathrm{yr} \\
(\%)\end{array}$ & $\begin{array}{l}>18 \mathrm{yr} \\
(\%)\end{array}$ \\
\hline 01 & 468 & $21.0 \%$ & 44 & 44 & 40 & 42 & 26 & 4 \\
\hline 02 & 450 & $28.0 \%$ & 53 & 52 & 49 & 49 & 25 & 11 \\
\hline 03 & 365 & $26.0 \%$ & 52 & 52 & 54 & 37 & 28 & 10 \\
\hline 04 & 381 & $23.0 \%$ & 59 & 61 & 40 & 21 & 11 & 7 \\
\hline 05 & 367 & $24.0 \%$ & 56 & 49 & 56 & 41 & 22 & 6 \\
\hline 06 & 363 & $25.0 \%$ & 68 & 57 & 44 & 36 & 15 & 6 \\
\hline 07 & 370 & $24.0 \%$ & 55 & 57 & 48 & 38 & 12 & 7 \\
\hline 08 & 328 & $27.0 \%$ & 68 & 54 & 50 & 33 & 25 & 9 \\
\hline 09 & 335 & $25.0 \%$ & 51 & 41 & 63 & 41 & 17 & 9 \\
\hline 10 & 341 & $23.0 \%$ & 50 & 45 & 48 & 33 & 24 & 8 \\
\hline
\end{tabular}

Results: See table

Conclusions: The carriage rates of pneumococci in UK families were higher than expected across all ages with no seasonal variation. No serotypes 1 \& 5 (present in the 9-valent vaccine) were isolated.

\section{G163 THE EPIDEMIOLOGICAL AND CLINICAL FEATURES OF CHRONIC GRANULOMATOUS DISEASE IN THE UK AND IRELAND}

L.K.R. Jones', P.J. McGrogan', A.J. Cant' ${ }^{2}$ L. Morton ${ }^{3}$, D. Goldblatt ${ }^{3}$, A. Thrasher $^{3}$, L. Parker ${ }^{3}$, T.J. Flood' ${ }^{2}$ 'Child Health, University of Newcastle, Newcastle upon Tyne; 'Department of Paediatric Immunology, NGH, Newcastle; ${ }^{3}$ Institute of Child Health, Great Ormond Street, London

Background: Many advances have been made in the management of Chronic Granulomatous Disease (CGD) and registries have been established world wide. Little was known however of the extent of the disease in the British Isles. Therefore, in 2000, a collaborative project, funded by the CGD Research Trust, established a registry in the UK and Ireland.

Aims: To delineate the incidence and clinical characteristics of CGD.

Methods: 1700 Consultants were contacted to identify patients. 1341 replied, identifying 115 patients: $92(80 \%)$ have so far consented. Comprehensive data on genetic and family history, clinical features, complications and treatments were abstracted from medical records.

Results: Birth prevalence was calculated as 1:147,000 $195 \% \mathrm{Cl}$ $1: 95,000-1: 241,000)$. There were 72 kindred, 65 with known inheritance. Of the total number of patients $66(72 \%)$ had XL CGD (median age of diagnosis 2.15 years, range $0.0-23.6) ; 16(17 \%)$ had $A R$ CGD (median age at diagnosis 16.6 years, range 0.9-51.1.) Comparison of observed versus expected cases registered, suggested up to $35 \%$ of patients did not survive beyond 30 years of age. Infections were common and often recurrent. They included: abscesses $(60 \%$ of patients), pneumonia $(47 \%)$, suppurative adentitis $(34 \%)$, septicaemia $(23 \%)$, osteomyelitis (12\%). Aspergillus spp were the commonest group of organisms isolated in pneumonia, but overall Staphyloccal spp were the most prevalent family. $53 \%$ of patients had gastrointestinal complications, $36 \%$ of who had colitis. All patients were taking some form of chemoprophylaxis: antibiotic (100\%), antifungal $(90 \%)$. Other treatments included steroids, interferon gamma, white cell transfusions and growth colony stimulating factor. 11 patients had a BMT and 1 had gene therapy.

Conclusion: The prevalence of CGD is higher than previously thought. Infections remain serious and common.

\section{G164 CHILDREN WITH HUMAN IMMUNODEFICIENCY VIRUS ADMITTED TO A PAEDIATRIC INTENSIVE CARE UNIT IN THE UNITED KINGDOM OVER A 10-YEAR PERIOD}

S. Cooper, E.G.H. Lyall, S. Walters, G. Tudor-Williams, P. Habibi, C. de Munter, J. Britto, S. Nadel. Paediatric Intensive Care Unit, St Mary's Hospital, Praed Street, London W2 INY

Introduction: There is limited experience of the management of children with human immunodeficiency virus (HIV) infection on a paediatric intensive care unit (PICU). There have been reports from South Africa showing a varied outcome with high mortality. There are no reported studies looking at this group in the United Kingdom, although data have been published on children with HIV and Pneumocystis carinii pneumonia (PCP) and cytomegalovirus (CMV) infection in the UK. We describe our experience of the management of children with HIV infection over a 10-year period.

Method: We performed a retrospective analysis of all children with HIV infection admitted to our PICU between August 1992 and July 2002. Their ages ranged from 2 months to 11 years. Information collected included demographic data, clinical presentation, investigations, treatment and outcome (short and long term).

Results: The total number of patients admitted to PICU with HIV during the study period was 42 , with 66 admission episodes. The number of patients that died in PICU was $16(38.1 \%)$, and that survived their last PICU admission was $26(61.9 \%)$. Of these, 5 $(19.2 \%)$ died at a later date (between 1 and 13 months after discharge from PICU) and $21(80.8 \%)$ survived to the time of reporting. The most frequent reasons for PICU admission were PCP $145.4 \%$ of all admissions) and respiratory failure due to other causes (31.8\%). There was evidence of CMV infection in 28 patients $(66.7 \%)$. Most of the current survivors had good outcomes in terms of growth and development, with $85.7 \%$ having normal growth and normal or mildly delayed development.

Conclusion: We have shown that although there is still a significant mortality among children with HIV admitted to PICU, many of them are surviving their admission and over $80 \%$ of the current survivors have good outcomes with the currently available highly active anti-retroviral therapy (HAART). This provides justification that intensive care treatment is appropriate for this group of patients in the United Kingdom

\section{G165 CHANGES IN VERTICALLY ACQUIRED PAEDIATRIC HIV IN UK AND IRELAND OVER CALENDAR TIME: THE COLLABORATIVE HIV PAEDIATRIC SURVEILLANCE (CHIPS) STUDY AND NATIONAL STUDY OF HIV IN PREGNANCY AND CHILDHOOD (NSHPC)}

K. Doerholt, T. Duong, M. Sharland, P. Tookey, J. Masters, D.M. Gibb on behalf of CHIPS and NSHPC. Medical Research Council and Institute of Child Health, London

Introduction: In the UK and Ireland, Highly Active Antiretroviral Therapy (HAART) and reduction of mother to Child Transmission (MTCT) has changed the epidemiology of paediatric HIV infection.

Aims: To describe changes in demography, clinical characteristics and disease progression of children infected through MTCT in UK and Ireland.

Methods: Data on clinical events and ART were collected on children alive and in followed up since 1996 at 16 CHIPS centres (about $70 \%$ of all vertically infected children ever reported in UK and Ireland). Demographic and further data on 86 additional children who died before 1996 were available from NSHPC.

Results: 685 children were infected through MTCT and $27 \%$ are older then 10 years. 490 under follow-up in 2001/02 compared to 305 in 1996. The proportion of newly presenting children born abroad has risen from $28 \%$ during $1992-99$ to $56 \%$ in $2000-02.334$ children developed one or more first AIDS diagnoses or died, 136 $(40 \%)$ within one month of presentation with HIV. Rates of first AIDS diagnoses decreased 5 -fold over calendar time $(13.2,9.1,7.5,4.0$, 6.1 and 2.4 / child-years-at-risk before-1997, $1997,1998,1999$ 2000 , and 2001/02), mirroring increased use of HAART. Of 147 
children progressing to AIDS/death since 1997, 125 were born to mothers with unknown HIV status. After 1997, $87 \%$ of children had opportunistic infection (OI) as one of their first AIDS diagnoses compared with 55\% before 1997.

Conclusion: The number of HIV infected children in follow up is still rising and many are entering adolescence. Although progression to AIDS has decreased 5-fold since pre-1997, recently presenting children are often born abroad and have late-stage disease, particularly Ols.

\section{G166 DIRECT AND INDIRECT INFECTION OF MONOCYTES WITH RSV INCREASES CHEMOKINE RECEPTOR EXPRESSION}

P.T. Morrison', J. Handforth', J. Uddin', M. Sharland', J.S. Friedland'. Dept. of Infectious Diseases, Faculty of Medicine, Imperial College of Science, Technology \& Medicine, Hammersmith Hospital, Du Cane Road, London W12 ONN, UK; ${ }^{2}$ Paediatric Infectious Diseases Unit, St George's Hospital, London SW17 ORE, UK

Aims: RSV infection is the most common cause of infant hospital admission in the UK. Serious lower respiratory tract infection is characterised by a large influx of leukocytes into the lung. This influx is regulated by chemokines, which are known to be upregulated in response to RSV infection both in vivo and in vitro. Here we examine the effect of RSV infection on chemokine receptor expression in human monocytic cells.

Results and Methods: Buffy coat derived monocytes, or 5-day matured Monocyte Derived Macrophages (MDM) were infected with RSV-A2 strain. CCR 1, 2 and 5 gene and protein expression was assessed by RNAase Protection Assay and FACS. Cytokine networks were investigated by exposing cells to RSV-CM \{conditioned media from respiratory epithelial A549 cells infected with RSV\}.

Monocytes were initially CD $14^{\text {tve }}, C C R 1^{\text {ve }}, 2^{\text {tve }}, 5^{\text {ve }}$. CCR2 expression diminished over $12 \mathrm{hrs}$ culture and was absent on MDMs. After direct RSV infection of monocytes, CCR 1, 2 and 5 expression was up-regulated at $24 \mathrm{hrs}$ and lost by $48 \mathrm{hrs}$. Treatment of monocytes with RSV-CM induced a similar pattern of CCR 1 expression but at $96 \mathrm{hrs}$ post exposure, CCR 1, 2 and 5 expression was again transiently up-regulated. Although MDMs had different basal levels of CCR expression, direct infection or RSV-CM also induced cycles of transient CCR expression on these cells. Using the inhibitors cytochalasin, cholchicine, actinomycin and cycloheximide it was demonstrated that the CCR cycling described requires both de novo protein synthesis and a functional cytoskeleton.

Conclusions: These data show that RSV up-regulates not only chemokines but also their cognate receptors; potentially exacerbating the inflammatory response caused by RSV infection.

\section{G167 REDUCTION IN NUMBER AND SEVERITY OF NUT ALLERGY REACTIONS BY INTERVENTION WITH A MANAGEMENT PLAN IN 608 NUT-ALLERGIC CHILDREN}

A.T. Clark, P.W. Ewan. Department of Paediatrics, Department of Allergy, Addenbrookes Hospital, Cambridge

Introduction: Peanut allergy is common ( 1 in 70 children) and the most frequent cause of fatal reactions to foods. Advice is poor; further reactions are common and deaths occur. We evaluated a management programme providing advice on nut avoidance and emergency medication.
Methods: 608 children with confirmed peanut or tree nut allergy seen in a single regional allergy centre. Male: female 1:1; Age $0-<5 y$ $42 \% ; 5-<10$ y $37 \%, 10-<16$ y $21 \%$. Severity of nut allergy was graded 1-5 and emergency medication allocated accordingly: oral antihistamine +/-inhaled and/or injected epinephrine (Epipen). Patients/ parents/school staff received detailed verbal \& written nut avoidance advice as well as training in recognition \& self-treatment of reactions, with a written treatment plan. At regular follow up over 22,907 patient-months (median $35 \mathrm{~m}$ ) retraining was given and details of further reactions obtained. Results: $130 / 608(21 \%)$ patients had a follow up reaction and this was of reduced severity compared to their worst reaction pre-referral. 98/130 (88\%) were mild (cutaneous only): 73 required oral antihistamine, 5 inhaled epinephrine and 17 no treatment. $28 / 130(21 \%)$ had a moderate follow up reaction (wheeze) all improved after self medication. Only 1/608 (0.16\%) had a severe follow up reaction (with dyspnoea) compared to $4 \%$ for the worst reaction before referral. Overall 12/608 received inhaled epinephrine (always effective) and 3/608 received self-injected epinephrine (ages 18,12 \& 11 y) which was always effective. Asthma was common $(57 \%$ of 608$)$ but well controlled.

Conclusions: $79 \%$ of patients had no further reactions compared to $45 \%$ in other series. The substantial reduction in incidence \& severity emphasises the importance of a management plan and repeated advice on nut avoidance. Self-treatment was effective (inhaled epinephrine for early laryngeal oedema and Epipen for severe reactions) but provision of this, including who should carry epinephrine, required assessment of allergy severity. We have demonstrated that this approach improves outcome and provided an evidence base for practice.

\section{G168 PAEDIATRIC MALARIA IN EAST LONDON}

S. Ladhani, H. El Bashir, V.S. Patel, D. Shingadia. Newham General Hospital and the Royal London Hospital

Objectives: To describe the epidemiological, clinical and laboratory features of children < 16 years of age with malaria in East London.

Methods: Paediatric malaria cases were identified retrospectively between 1995-2000 using notifications and hospital discharge data.

Results: A total of 211 children with a median age of 9 years (range 11-179 months) were identified. Imported malaria accounted for $82 \%$ of cases while the rest were children visiting from endemic areas. Three quarters of children with imported malaria were born in the UK and $93 \%$ were of Black African ethnicity. The peak seasonal incidence was late summer/early autumn. Plasmodium falciparum acquired in Africa accounted for $91 \%$ of cases. Although $41 \%$ of children took antimalarial prophylaxis, only $15 \%$ were taken according to recommended guidelines. Another family member, most often a sibling, was found to have concurrent malaria in $23 \%(49 / 211)$. Of 114 children seen first by their GP, malaria was suspected in $32 \%$. In contrast, malaria was suspected in $89 \%$ of children initially presenting to casualty. Seventy one percent of cases had parasitaemia less than $2 \%$ and only 5 patients had levels $>10 \%$. Nine children $(4.3 \%)$ had complicated malaria based on World Health Organization classification ( 6 cerebral malaria, 3 renal failure). All children responded to antimalarial therapy, with no reported deaths.

Conclusions: The majority of children with imported malaria were UK-born, school-aged, of Black African ethnicity, and were visiting family in Africa, often with other family members. Most children had low-level parasitaemia and uncomplicated malaria. We suggest that this population be targeted for future interventions to prevent malaria. Furthermore, we propose that screening of other travelling family members may be justified to exclude malaria co-infection. 